\title{
Hyperprolactinemia in Acromegaly is Related to Prolactin Secretion by Somatolactotroph Tumours
}

\author{
Authors \\ Delphine Van Laethem ${ }^{1}$, Alex Michotte ${ }^{2}$, Wilfried Cools ${ }^{3}$, Brigitte Velkeniers ${ }^{1}$, David Unuane ${ }^{1}$, C. E. Andreescu ${ }^{1}$, \\ Bert Bravenboer ${ }^{1}$
}

\begin{abstract}
Affiliations
1 Endocrinology, Universitair Ziekenhuis Brussel, Brussel, Belgium

2 Department of Pathology (Neuropathology), Universitair Ziekenhuis Brussel, Brussel, Belgium

3 Statistics, Vrije Universiteit Brussel, Brussel, Belgium
\end{abstract}

Key words

anterior pituitary, prolactin, GH-Prl adenoma, pituitary tumour, somatolactotroph tumour

$\begin{array}{ll}\text { received } & 01.11 .2019 \\ \text { accepted } & 17.06 .2020\end{array}$

Bibliography

DOI https://doi.org/10.1055/a-1207-1132

Published online: 5.8.2020

Horm Metab Res 2020; 52: 647-653

(c) Georg Thieme Verlag KG Stuttgart · New York

ISSN 0018-5043

Correspondence

Delphine Van Laethem

Endocrinology, Universitair Ziekenhuis Brussel

Laarbeeklaan 101

1090 Brussel

Belgium

Tel.: + 322477 6418, Fax: + 3224776428

delphine.van.laethem@vub.be;

delphinevanlaethem@gmail.com

\section{ABSTRACT}

The aim of this study is to assess differences in patient characteristics, tumour characteristics and hormone levels between acromegalic patients with and without hyperprolactinemia. 44 patients of the University Hospital of Brussels, Belgium with acromegaly who were diagnosed between January 2007 and July 2018 were included in this study. Nineteen patients were classified in the hyperprolactinemia group and 25 patients were classified in the normoprolactinemia group. No significant differences between acromegalic patients with and without hyperprolactinemia were found in age at diagnosis, gender, presence of hyperprolactinemia symptoms, insulin-like growth factor 1, growth hormone and testosterone levels, tumour volume, tumour invasiveness, immunohistochemistry of growth hormone and prolactin, $\mathrm{Ki}-67$ index and mitotic index. However, for a cut-off of $10 \%$ of prolactin-positive cells, there was a trend towards a higher percentage of prolactin-positive tumours in hyperprolactinemia patients $(p=0.054)$ and higher mean prolactin level in case of positive prolactin immunostaining $(p=0.007))$. In our study there were no differences in characteristics between acromegaly patients with hyper- and normoprolactinemia. An association between the serum prolactin level and the positivity of prolactin immunohistochemistry of the adenoma tissue was found. The absence of a difference in tumour volume between patients with hyper- and normoprolactinemia suggests that the hyperprolactinemia is likely to be caused by the co-secretion of growth hormone and prolactin by the tumour. Finally, for the first time, the cut-off of $10 \%$ of prolactin cells was validated for the diagnosis of somatolactotroph tumours in acromegaly.

\section{Introduction}

Acromegaly is a rare disease associated with numerous co-morbidities, not all of which are reversible after treatment, and a higher mortality rate [1-3]. In about $20-35 \%$ there is an elevated level of prolactin (PRL), either caused by secretion of PRL by the tumour or by pituitary stalk impingement by the tumour mass [4-10].

There are several studies concerning the prognostic value of serum PRL and a positive PRL immunostaining in the tumour of patients with acromegaly [11-14], as well as a positive correlation between preoperative PRL levels and tumour size, suprasellar tumour extension and invasive growth of the adenoma [9].
The aim of the present study is to evaluate whether there are differences between patients with acromegaly and patients with acromegaly and hyperprolactinemia, including patients who received different treatments. In this study patient characteristics, hormone levels (IGF-1, GH after oral glucose tolerance test (OGTT), PRL and in male patients testosterone), clinical presentation, tumour size and immunostaining of $\mathrm{GH}$ and PRL were compared between these two groups. Furthermore, invasiveness on MRI, the Ki-67 labelling index and the mitotic index, which are recommended parameters to assess clinical aggressiveness of pituitary tu- 
mours, were analysed [15]. Possible differences in these parameters could have implications for diagnostic and treatment strategies for patients with acromegaly with or without hyperprolactinemia specifically.

\section{Patients and Methods}

\section{Patient selection and characteristics}

This retrospective cross-sectional study was carried out by analysing the data collected from the patient files of the University Hospital of Brussels, Vrije Universiteit Brussel (VUB), Belgium. The diagnosis of acromegaly was based on an elevated IGF-1 level and a $\mathrm{GH}$ level of more than $1 \mu \mathrm{g} / \mathrm{l}$ following an oral glucose tolerance test [16]. Patients were only included in this study if they had been diagnosed with acromegaly between January 2007 and July 2018 and if a PRL level was known before the start of treatment. This last criterion excluded only three patients.

In total, 44 patients met the criteria of inclusion. These 44 patients were then divided into two groups, based on the PRL level before the start of treatment: 19 patients had an elevated PRL level and were classified in the acromegaly with hyperprolactinemia group (Group 1) and 25 patients had a normal PRL level and were classified in the acromegaly group (Group 2). Forty-two of those patients received surgery at one point in their treatment and of those 42 patients, four received medical therapy beforehand.

Besides gender and age at diagnosis, the presence of symptoms that could be compatible with hyperprolactinemia at the time of diagnosis was assessed. These symptoms include oligo- or amenorrhea, galactorrhea, infertility, decreased libido and impotence [8].

This study was approved by the Medical Ethics Committee of the University Hospital of Brussels, VUB, Belgium.

\section{Hormone levels}

As stated above, the diagnosis of acromegaly is based on an elevated IGF-1 level, as matched for age and gender, and insufficient suppression of GH after an OGTT. An abnormal OGTT was defined as a $\mathrm{GH}$ level of more than $1 \mu \mathrm{g} / \mathrm{l}$ two hours after $75 \mathrm{~g}$ of oral glucose.

In the University Hospital of Brussels, VUB, where most of the analyses were carried out, IGF-1 was measured in serum using a chemiluminescence immunoassay (iSYS/IDS). GH was also measured using a chemiluminescence immunoassay (iSYS/IDS). The first IGF-1 value and GH value after OGTT before the start of the treatment were used (i. e., for IGF-1 19 patients in Group 1 and 25 in Group 2, for GH after OGTT 8 in Group 1 and 18 in Group 2). Since there is significant variation in laboratory results of IGF- 1 and $\mathrm{GH}$ between different laboratories $[17,18]$, values measured in the laboratory of the University Hospital of Brussels, VUB, were used if possible (i. e., for IGF-1 14 patients in Group 1 and 15 in Group 2, for GH after OGTT 3 in Group 1 and 11 in Group 2). If no pre-treatment values from the reference laboratory were known, values from other laboratories were assessed.

Prolactin was measured in serum using electrochemilumeniscence (Cobas 8000/Roche Diagnostics). The reference ranges were
$4.04-15.02 \mu \mathrm{g} / \mathrm{l}$ in men above 17 years of age and $4.79-23.30 \mu \mathrm{g} / \mathrm{l}$ in women older than 17. The first PRL value after the diagnosis of acromegaly but before the start of the treatment was used (i.e., 19 patients in Group 1 and 25 in Group 2). In male subjects we also looked at total testosterone levels at the time of diagnosis. This was measured using electrochemilumeniscence (Cobas 8000/Roche Diagnostics). The reference ranges were $2.50-8.40 \mu \mathrm{g} / \mathrm{l}$ between the ages of 18 and 49 and between 1.90 and $7.40 \mu \mathrm{g} / \mathrm{l}$ in patients older than 50 .

\section{Tumour characteristics}

Tumours were classified as macroadenomas if the tumour diameter was larger than $10 \mathrm{~mm}$ [8]. Tumour volume was calculated based on the diameters of the tumour measured on MRI. The geometric formula: $1 / 2 \times$ (length $\times$ width $\times$ height), was used to calculate the volume, which is a sufficiently accurate estimation of tumour volume in most cases $[19,20]$. The first MRI before the start of the treatment in which length, width and height were known, was used for the measurements (i.e., 13 patients in Group 1 and 16 in Group 2). Tumour invasiveness was assessed by radiologists by evaluating MRI images (i. e. 19 patients in Group 1 and 25 in Group 2) [21].

Pathological examination of the pituitary tumour obtained by transsphenoidal surgery (TSS) was carried out in the department of Pathology by the same neuropathologist (Prof. Dr. A. Michotte), in the University Hospital of Brussels, VUB. The specimens were fixed in a $10 \%$ formalin solution and embedded in paraffin. Staining was performed with the hematoxylin-eosin method. Immunohistochemistry (IHC) was performed according to standard methods using antibodies against GH and PRL (GH Ventana Roche Catalogue number 760-2804 - polyclonal and PRL Ventana Roche - Catalogue number 760-2803 - polyclonal) and positive cells were counted optically. Tumours were classified as somatotroph adenomas if they stained positive for $\mathrm{GH}$ and, according to Villa et al. [22], classified as somatolactotroph tumours if there is both positive staining for $\mathrm{GH}$ and at least $10 \%$ of the cells for PRL (see $>$ Figs. 1 and $\mathbf{2}$ for an example of an adenoma with respectively $<10 \%$ and $\geq 10 \%$ PRL-positive cells). The IHC of PRL was also analysed for two other cut-offs for PRL-positive cells, namely a cut-off of $\geq 5 \%$ and scattered PRL-positive cells. The distinction between sparsely and densely granulated adenomas was not analysed and no distinction was made between somatolactotroph and the rare mammosomatotroph tumours, with cosecretion of $\mathrm{GH}$ and PRL by the same cells.

The Ki-67 labelling index was calculated by IHC with antibodies against Ki-67 (Ki-67 Ventana Roche - Catalogue number 790-4286 - monoclonal clone 30-9) and optically counting the positive cells and was considered elevated if more than $3 \%$ of the nuclei stained positive (i. e., 13 patients in Group 1 and 20 in Group 2) [15]. The mitotic index was calculated by counting mitoses on hematoxylin-eosin staining (i. e., 17 patients in Group 1 and 23 in Group 2).

\section{Statistics}

Statistical analysis was performed using IBM SPSS Statistics, version 23.0 software. Data were either normally distributed or trans- 
formed to be normally distributed using logarithmic transformation. Results were reported as means with $95 \%$ confidence intervals $(95 \% \mathrm{Cl})$ or as percentages. When a transformation was carried out, the back-transformed means and confidence intervals were reported. Differences in the means between the two groups were assessed using the Student's unpaired $t$-test for the continuous variables. For the discrete variables differences were assessed using the Fisher's Exact test. The predictive factors for hyperprolactine-

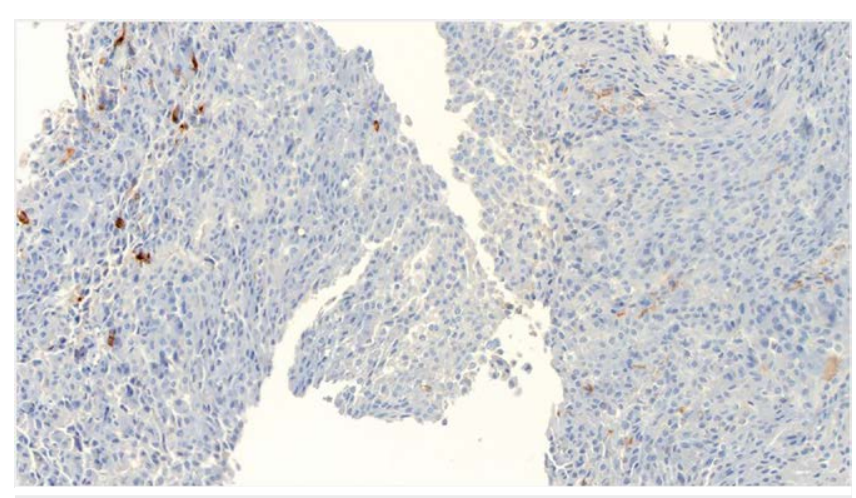

- Fig. 1 Example of a somatotroph tumour with scattered PRL-immunoreactive cells.

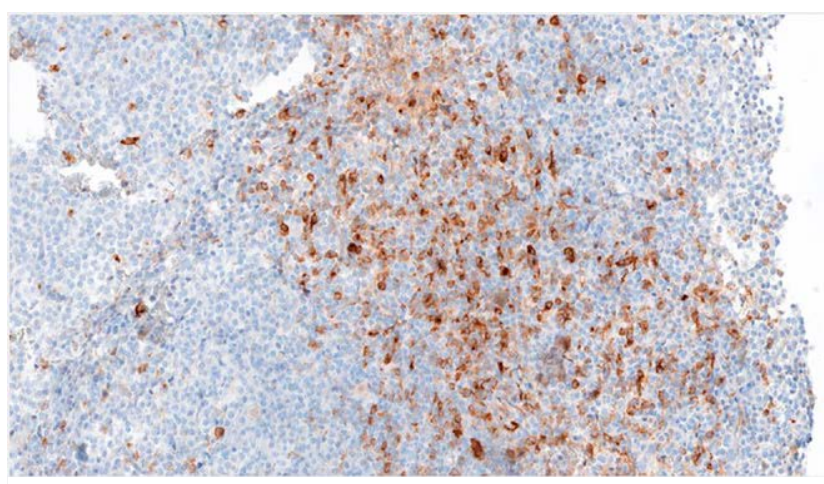

Fig. 2 Example of a somatolactotroph tumour with $\geq 10 \%$ PRL-immunoreactive cells. mia were determined by logistic regression. A two-sided test with a type I error probability of 0.05 was used.

\section{Results}

\section{Patient characteristics}

In the acromegaly with hyperprolactinemia group (Group 1), which consisted of 19 patients, the mean age at diagnosis was 45.4 years [95\% Confidence Interval (Cl): 37.2-53.7] and 52.6\% were females. In $31.6 \%$ of the cases symptoms compatible with hyperprolactinemia were recorded at presentation. In the acromegaly group (Group 2), which consisted of 25 patients, the mean age was 49.5 years ( $95 \% \mathrm{Cl}: 44.3-54.7$ ), $60 \%$ of the patients were female and $20 \%$ had symptoms of hyperprolactinemia. There were no significant differences in age at diagnosis, gender or the presence of symptoms associated with hyperprolactinemia between the two groups $(p=0.370, p=0.761$, and $p=0.489$, respectively).

\section{Hormone levels}

The mean hormone levels of the two groups are listed in $>$ Table 1. There was a significant difference in the mean PRL level of the two groups. Differences in IGF-1 and GH levels, both when only measurements carried out in the reference laboratory were included and when all measurements were included were not statistically significant (see $>$ Table 1). There was no correlation between hormone levels and tumour volume (IGF-1: $p=0.061, \mathrm{GH}: \mathrm{p}=0.056$, PRL: $p=0.667)$. In male subjects, the difference in total testosterone levels was not statistically significant.

\section{Tumour characteristics}

In $>$ Table 2, the percentage of macroadenomas, tumour volume, tumour invasiveness, IHC of GH and PRL, elevated Ki-67 labelling index and mitotic index are listed. No significant differences in these parameters between the two groups were detected, albeit the difference in \% of PRL-positive adenomas (if the cut-off for positivity is $\geq 10 \%$ PRL-positive cells was used) was nearly statistically significant ( $p=0.054)$, and there was no difference in the IHC of $\mathrm{GH}$ and the mitotic index. Of note, the highest \% of PRL-positive cells in a tumour was $90 \%$, which was seen in four adenomas, of which only two also showed an elevated serum PRL level.

- Table 1 Hormone levels in Group 1 and 2.

\begin{tabular}{|c|c|c|c|}
\hline & Group 1: Acromegaly + hyperprolactinemia & Group 2: Acromegaly & p-Value \\
\hline IGF-1 & $754.05 \mu \mathrm{g} / \mathrm{l}(95 \% \mathrm{Cl}: 612.63-928.11)$ & $682.34 \mu \mathrm{g} / \mathrm{l}(95 \% \mathrm{Cl}: 586.20-819.60)$ & 0.458 \\
\hline IGF-1 UZB & $762.96 \mu \mathrm{g} / \mathrm{l}(95 \% \mathrm{Cl}: 588.30-989.46)$ & $601.59 \mu \mathrm{g} / \mathrm{l}(95 \% \mathrm{Cl}: 468.71-772.15)$ & 0.167 \\
\hline $\operatorname{OGTT}(G H)$ & $13.21 \mu \mathrm{g} / \mathrm{l}(95 \% \mathrm{Cl}: 3.48-50.05)$ & $4.98 \mu \mathrm{g} / \mathrm{l}(95 \% \mathrm{Cl}: 2.33-10.65)$ & 0.151 \\
\hline OGTT UZB (GH) & $14.34 \mu \mathrm{g} / \mathrm{l}(95 \% \mathrm{Cl}: 0.02-8978.42)$ & $3.77 \mu \mathrm{g} / \mathrm{l}(95 \% \mathrm{Cl}: 1.24-11.42)$ & 0.287 \\
\hline PRL & $54.57 \mu \mathrm{g} / \mathrm{l}(95 \% \mathrm{Cl}: 29.49-101.00)$ & $9.36 \mu \mathrm{g} / \mathrm{l}(95 \% \mathrm{Cl}: 7.49-11.70)$ & 0.000 \\
\hline Testosterone & $2.64 \mu \mathrm{g} / \mathrm{l}(95 \% \mathrm{Cl}: 1.64-3.63)$ & $2.92 \mu \mathrm{g} / \mathrm{l}(95 \% \mathrm{Cl}: 1.78-4.06)$ & 0.649 \\
\hline
\end{tabular}

UZB: Measurements carried out at the University Hospital of Brussels. OGTT: GH level two hours after OGTT. 


\section{Immunohistochemistry prolactin - Prolactin level}

The relationship between PRL IHC and the PRL levels was also assessed, regardless of the Group in which they were included. In - Table 3, the mean levels of PRL in the presence or absence of a positive PRL IHC, with cut-offs for positivity of $\geq 10 \%, \geq 5 \%$ and scattered cells respectively, are listed. Changing the cut-off for PRL-positivity evidently changed the number of PRL-positive tumours: A cut-off of $\geq 10 \%$ resulted in 20 positive and 19 negative tumours, a cut-off of $\geq 5 \%$ in 24 positive and 15 negative tumours and a cut-off scattered cells in 32 positive and 7 negative tumours.

There is a statistically higher mean PRL level in the presence of a positive PRL IHC when the cut-offs of $\geq 10 \%$ or $\geq 5 \%$ are used ( $p=0.007$ and $p=0.024$, respectively). The difference is not statistically significant with the cut-off of scattered cells $(p=0.244)$. Of note, 2 out of 10 tumours with no PRL-positive cells, both macroadenomas, still had an elevated serum PRL level and the correlation between the percentage of PRL-positive cells and tumour volume was not analysed.

\section{Logistic regression}

A logistic regression was performed to attempt to predict the likelihood of hyperprolactinemia in the presence of different predictive factors. Age at diagnosis and gender were included as predictors to control for these factors. Tumour invasiveness and the Ki-67 labelling index were included to control for tumour aggressiveness. The presence of symptoms of hyperprolactinemia was added to take clinical presentation into account and PRL IHC was included to allow for assessment of secretion of PRL by the tumour.

The logistic regression model was statistically significant $\left[X^{2}(6)=\right.$ 12.864, $p=0.045$ ]. The model explained $43.7 \%$ (Nagelkerke $R^{2}$ ) of the variance in hyperprolactinemia and correctly classified $78.8 \%$ of the cases. Hyperprolactinemia was 19.257 times more likely

- Table 2 Tumour characteristics in Group 1 and 2.

\begin{tabular}{|c|c|c|c|}
\hline & $\begin{array}{l}\text { Group 1: Acromegaly + hyperprolac- } \\
\text { tinemia }\end{array}$ & Group 2: Acromegaly & p-Value \\
\hline PRL & $54.57 \mu \mathrm{g} / \mathrm{l}(95 \% \mathrm{Cl}: 29.49-101.00)$ & $9.36 \mu \mathrm{g} / \mathrm{l}(95 \% \mathrm{Cl}: 7.49-11.70)$ & 0.000 \\
\hline Macroadenomas & $89.5 \%$ & $72 \%$ & 0.260 \\
\hline Tumour volume & $\begin{array}{l}2320.60 \mathrm{~mm}^{3} \\
(95 \% \mathrm{Cl}: 904.07-5956.62)\end{array}$ & $\begin{array}{l}1563.51 \mathrm{~mm}^{3} \\
\text { (95\% Cl: } 791.41-3088.87)\end{array}$ & 0.460 \\
\hline Invasive on MRI & $63.2 \%$ & $40 \%$ & 0.223 \\
\hline IHC GH & $100 \%$ & $100 \%$ & NA \\
\hline IHC PRL $\geq 10 \%$ & $70.6 \%$ & $36.4 \%$ & 0.054 \\
\hline IHC PRL $\geq 5 \%$ & $76.5 \%$ & $50 \%$ & 0.112 \\
\hline IHC PRL scattered cells & $88.2 \%$ & $77.5 \%$ & 0.438 \\
\hline Ki-67 labelling index elevated & $15.4 \%$ & $5 \%$ & 0.547 \\
\hline Mitotic index & $0 \%$ & $0 \%$ & NA \\
\hline
\end{tabular}

IHC PRL $\geq 10 \%$ : Cut-off of at least $10 \%$ PRL-immunoreactive cells; IHC PRL $\geq 5 \%$ : Cut-off of at least $5 \%$ PRL-immunoreactive cells; IHC PRL scattered: Cut-off of at least scattered immunoreactive cells. NA: Not available.

- Table 3 Comparison of cut-offs of PRL IHC positivity of the tumour and the serum PRL levels.

\begin{tabular}{|c|c|c|c|}
\hline \multirow[b]{2}{*}{ Cut-off for PRL-positivity } & \multicolumn{3}{|c|}{ Serum PRL levels } \\
\hline & PRL IHC positive $\left(\mathbf{n}^{+}\right)$ & PRL IHC negative ( $\left.n^{-}\right)$ & p-Value \\
\hline IHC PRL $\geq 10 \%\left(n^{+}=20, n^{-}=19\right)$ & $24.87 \mu \mathrm{g} / \mathrm{l}(95 \% \mathrm{Cl}: 16.71-36.99)$ & $11.69 \mu \mathrm{g} / \mathrm{l}(95 \% \mathrm{Cl}: 7.91-17.28)$ & 0.007 \\
\hline IHC PRL $\geq 5 \%\left(n^{+}=24, n^{-}=15\right)$ & $22.21 \mu \mathrm{g} / \mathrm{l}(95 \% \mathrm{Cl}: 15.03-32.83)$ & $11.45 \mu \mathrm{g} / \mathrm{l}(95 \% \mathrm{Cl}: 7.67-17.10)$ & 0.024 \\
\hline IHC PRL scattered cells $\left(n^{+}=32, n^{-}=7\right)$ & $18.65 \mu \mathrm{g} / \mathrm{l}(95 \% \mathrm{Cl}: 13.18-26.38)$ & $11.96 \mu \mathrm{g} / \mathrm{l}(95 \% \mathrm{Cl}: 7.83-18.27)$ & 0.244 \\
\hline
\end{tabular}


(95\% Cl: 1.926-192.524, $\mathrm{p}=0.012$ ) when there was a positive PRL IHC (cut-off $\geq 10 \%$ ). The other predictors were not statistically significant: gender [Odds ratio $(\mathrm{OR})=0.368,95 \% \mathrm{Cl}: 0.053-2.538$, $\mathrm{p}=0.310]$, age at diagnosis $[\mathrm{OR}=0.988,95 \% \mathrm{Cl}: 0.915-1.067$, $\mathrm{P}=0.761$ ], [invasiveness on MRI [OR $=7.040$, $95 \% \mathrm{Cl}: 0.829-$ 59.749, $\mathrm{p}=0.074$ ], symptoms of hyperprolactinemia $[\mathrm{OR}=3.392$, $95 \% \mathrm{Cl}: 0.272-42.227, \mathrm{p}=0.343$ ] and $\mathrm{Ki}-67$ labelling index [OR $=1.650,95 \% \mathrm{Cl}: 0.130-208.467, \mathrm{p}=0.839]$. Other factors were one by one included in this model to test for significance: IGF-1, GH after an OGTT, tumour volume and whether or not the tumour is a macroadenoma. Since this did not result in statistically significant models and they were not statistically significant predictors of hyperprolactinemia, these variables were not included in the final model.

When the cut-offs of $\geq 5 \%$ or scattered cells for positivity of PRL IHC were used, the logistic regression model was no longer statistically significant $\left[X^{2}(6)=9.543, p=0.145\right.$ and $X^{2}(6)=3.554$, $\mathrm{P}=0.737$, respectively].

\section{Discussion and Conclusions}

In this study, no significant differences between acromegaly patients with and without hyperprolactinemia were found in mean age at diagnosis, gender, presence of hyperprolactinemia symptoms, mean IGF-1 and GH levels, mean testosterone levels in male patients, percentage of macroadenomas, mean tumour volume, tumour invasiveness, positivity of GH and PRL immunostaining [albeit for PRL the difference was nearly statistically significant $(p=0.054)$ ], Ki-67 labelling index and mitotic index. However, the mean PRL level was significantly higher in the presence of a positive PRL IHC for a cut-off for positivity of $\geq 5$ and $\geq 10 \%$ of the cells staining positive. Furthermore, in the logistic regression model PRL IHC with a positivity cut-off of $10 \%$ was the only significant predictor of hyperprolactinemia, with an odds ratio of 19.275.

The strength of this study is that it is the first to compare the characteristics of acromegalic patients with and without hyperprolactinemia, with patients selected from the total acromegaly population, as opposed to those solely having received surgical treatment. We expected more patients who received a treatment other than surgery since in a nationwide survey from June 2003 till September 2004 in Belgium, only $68 \%$ of the patients underwent surgical treatment [23]. This could be due to possible bias by selecting patients who were treated in a university hospital, or by a shift in treatment strategies since this survey was carried out. A second strength is that most studies assessing tumour size only made the distinction between micro- and macroadenoma or only assessed the maximal diameter. In this study, the tumour size was assessed more precisely by calcultating tumour volume using a geometric formula. Finally, another strength is that this study is the first to compare tumour invasivity, Ki-67 labelling index and mitotic count between acromegaly patients with and without hyperprolactinemia. These factors are recommended to be analysed by the fourth edition of the WHO Classification of Tumours of the Pituitary Gland to assess possible clinical aggressiveness of pituitary adenomas [15].
The most important weakness of this study is its small sample size, which is inherent to the rarity of acromegaly [1]. Furthermore, a potential source of bias is the fact that since this is a retrospective study, it is impossible to ascertain that the presence of symptoms compatible with hyperprolactinemia was sufficiently questioned in each patient included in this study. Finally, it is important to note that stress can elevate PRL levels [8]. It was impossible to assess this factor in retrospectively collected data.

Wang et al. studied the retrospectively collected data of 279 surgically treated acromegaly patients, 97 of whom had concomitant hyperprolactinemia. They found that patients with acromegaly and concomitant hyperprolactinemia were younger at diagnosis and had less features of acromegaly and more features of hyperprolactinemia. Furthermore, they found a lower $\mathrm{GH}$ level after OGTT in the hyperprolactinemia group. They also state that there was a larger mean maximal adenoma diameter and a higher percentage of macroadenomas in the presence of hyperprolactinemia. Finally, they found a higher percentage of tumours with a PRL-positive IHC in the hyperprolactinemia group. However, there was no correlation between the serum PRL level and the percentage of adenoma cells with a positive PRL IHC [14]. Our study found a higher mean serum PRL level in the presence of a positive PRL IHC and the difference between the percentage of PRL-positive adenomas between the hyperprolactinemia and normoprolactinemia group was nearly statistically significant $(p=0.054)$ when a cut-off of PRL-positivity of $\geq 10 \%$ was used. Other differences found by Wang et al. could not be confirmed.

This study found a higher mean PRL level in case of a positive PRL IHC (when the cut-off for positivity of $\geq 5 \%$ and $\geq 10 \%$ was used) and a positive PRL IHC (cut-off $\geq 10 \%$ ) turned out to be a significant predictor of hyperprolactinemia in the logistic regression model. While the hyperprolactinemia found in two of the PRL-negative adenomas was likely caused by compression of the hypothalamus by the tumour mass, which inhibits dopamine secretion and thereby promotes PRL secretion [24], both tumour volume and the percentage of macroadenomas were not significantly different between patients with hyper- and normoprolactinemia in our study. Our findings suggest that hyperprolactinemia in acromegaly patients is likely to be caused by co-secretion of PRL by the tumour and less likely by pituitary stalk compression and validate the cutoff of $\geq 10 \%$ PRL-positive cells as a criterion for the histological diagnosis of a somatolactotroph tumour, as proposed by Villa et al. [22]. While Trouillas et al. found a correlation between serum PRL levels and the percentage of cells with a positive PRL IHC [24], four other studies contradict this finding $[13,14,25,26]$. However, Kreutzer et al. found that patients with a positive IHC for both $\mathrm{GH}$ and PRL had higher serum PRL levels than those with only a positive GH IHC [12]. The findings of Kreutzer et al. are in line with the findings of our study. These studies suggest that although most studies found no correlation between the serum PRL level and the percentage of PRL-positive cells on IHC, there seems to be a higher PRL level in the presence of PRL-positive cells. This suggests that somatolactotroph tumours do secrete PRL but that a higher percentage of positive cells does not necessarily equal a higher PRL level. 
Hyperprolactinemia seems to be associated with lower remission rates for acromegaly. Moreover, there are studies suggesting a similar trend in $\mathrm{GH}$-secreting tumours with a positive PRL IHC. De Marinis et al. found higher pre- and postoperative PRL levels and a high percentage of positive PRL immunohistochemistry in patients with poorly controlled acromegaly [11]. A lower surgical control rate on MRI three months after surgery in the presence of preoperative hyperprolactinemia has also been described [14]. Similarly, Nyquist et al. observed a lower surgical success rate in patients with the GH-PRL immunostain tumour subtype [13]. Finally, Kreutzer et al. noted that patients with a positive PRL IHC had lower remission rates [12]. In our study it was not possible to evaluate remission rates, since the follow-up of several patients was carried out in other hospitals. Moreover, because of the retrospective character of the study there was insufficient uniformity regarding the definition of remission.

More research is needed to assess whether acromegaly with both hyperprolactinemia and a positive PRL IHC is associated with lower remission rates in comparison to acromegaly patients without or with only one of those characteristics and whether these patients might benefit from a different or more aggressive treatment. For example, perhaps it is indicated to immediately associate dopamine agonists in case of an elevated PRL level or positive PRL IHC. Dopamine agonists, such as cabergoline, are mainly used in the treatment of hyperprolactinemia but also have their place in the treatment of acromegaly. There they are currenlty used mainly in case of mildly elevated IGF-1 levels or a as adjuvant therapy to a treatment with a somatostatin receptor ligand or a $\mathrm{GH}$ receptor antagonist. Among other factors, baseline PRL concentration is related to IGF-1 decline and tumour shrinkage in cabergoline monotherapy and to better response to treatment with the combination of a $\mathrm{GH}$ receptor antagonist and cabergoline [27]. However, more research is needed to draw definite conclusions.

Another interesting research track could be to assess the percentage of sparsely and densely granulated adenomas in patients with normo- and hyperprolactinemia, since the sparsely granulated subtype is associated with a more aggressive biological phenotype that may not respond well to somatostatin analogues, possibly due to lower somatostatin receptor 2 (SSTR-2) expression $[15,28]$. Looking at the granulation and SSTR-2 expression in mixed $\mathrm{GH}-\mathrm{PRL}$ adenomas could therefore help us predict treatment response.

To conclude, in this retrospective study of 44 acromegalic patients there were no differences in characteristics between acromegaly patients with hyper- and normoprolactinemia. From our results, we conclude that hyperprolactinemia in acromegaly is often caused by tumour secretion and the cut-off $\geq 10 \%$ of PRL-positive cells for the diagnosis of somatolactotroph tumours is validated.

\section{Conflict of Interest}

The authors declare that they have no conflict of interest.
References

[1] Holdaway IM, Rajasoorya C. Epidemiology of acromegaly. Pituitary 1999; 2: 29-41

[2] Abreu A, Tovar AP, Castellanos R et al. Challenges in the diagnosis and management of acromegaly: A focus on comorbidities. Pituitary 2016; 19: 448-457

[3] Colao A, Ferone D, Marzullo P et al. Systemic complications of acromegaly: Epidemiology, pathogenesis, and management. Endocr Rev 2004; 25: 102-152

[4] Melmed S. Acromegaly pathogenesis and treatment. J Clin Invest 2009; 119: 3189-3202

[5] Trepp R, Stettler C, Zwahlen M et al. Treatment outcomes and mortality of 94 patients with acromegaly. Acta Neurochir (Wien) 2005; 147: 243-251

[6] Mortini P, Losa M, Barzaghi R et al. Results of transsphenoidal surgery in a large series of patients with pituitary adenoma. Neurosurgery 2005; 56: 1222-1233

[7] Evran M, Sert M, Tetiker T. Clinical experiences and success rates of acromegaly treatment: the single center results of 62 patients. BMC Endocr Disord 2014; 14: 97

[8] Melmed S, Jameson JL. Anterior Pituitary Tumor Syndromes. In: Kasper DL, Fauci AS, Hauser SL, Longo DL, Jameson JL, Loscalzo J, Ed Harrison's Principles of Internal Medicine. New York: McGraw-Hill Education; 2015: 2261-2274

[9] Voit D, Saeger W, Ludecke DK. Pituitary adenomas in acromegaly: Comparison of different adenoma types with clinical data. Endocr Pathol 1999; 10: 123-135

[10] Andersen M, Hagen C, Frystyk J et al. Development of acromegaly in patients with prolactinomas. Eur J Endocrinol 2003; 149: 17-22

[11] De Marinis L, Zuppi P, Valle D et al. A retrospective hormonal and immunohistochemical evaluation of 47 acromegalic patients: Prognostic value of preoperative plasma prolactin. Horm Metab Res 2002; 34: 137-143

[12] Kreutzer ], Vance ML, Lopes MB et al. Surgical management of $\mathrm{GH}$-secreting pituitary adenomas: An outcome study using modern remission criteria. J Clin Endocrinol Metab 2001; 86: 4072-4077

[13] Nyquist P, Laws ER Jr., Elliott E. Novel features of tumors that secrete both growth hormone and prolactin in acromegaly. Neurosurgery 1994; 35: 179-183. discussion 183-174

[14] Wang M, Mou C, Jiang M et al. The characteristics of acromegalic patients with hyperprolactinemia and the differences in patients with merely GH-secreting adenomas: Clinical analysis of 279 cases. Eur J Endocrinol 2012; 166: 797-802

[15] Mete O, Lopes MB. Overview of the 2017 WHO Classification of Pituitary Tumors. Endocr Pathol 2017; 28: 228-243

[16] Katznelson L, Laws ER Jr., Melmed S et al. Acromegaly: An endocrine society clinical practice guideline. J Clin Endocrinol Metab 2014; 99 : 3933-3951

[17] Arafat AM, Mohlig M, Weickert MO et al. Growth hormone response during oral glucose tolerance test: The impact of assay method on the estimation of reference values in patients with acromegaly and in healthy controls, and the role of gender, age, and body mass index. J Clin Endocrinol Metab 2008; 93: 1254-1262

[18] Frystyk J, Freda P, Clemmons DR. The current status of IGF-I - a 2009 update. Growth Horm IGF Res 2010; 20: 8-18

[19] Chuang CC, Lin SY, Pai PC et al. Different volumetric measurement methods for pituitary adenomas and their crucial clinical significance. Sci Rep 2017; 7: 40792

[20] Davies BM, Carr E, Soh C et al. Assessing size of pituitary adenomas: A comparison of qualitative and quantitative methods on MR. Acta Neurochir (Wien) 2016; 158: 677-683 
[21] Scheithauer BW, Kovacs KT, Laws ER et al. Pathology of invasive pituitary tumors with special reference to functional classification. J Neurosurg 1986; 65: 733-744

[22] Villa C, Vasiljevic A, Jaffrain-Rea ML et al. A standardised diagnostic approach to pituitary neuroendocrine tumours (PitNETs): A European Pituitary Pathology Group (EPPG) proposal. Virchows Archiv 2019; 475: 687-692

[23] Bex M, Abs R, T'Sjoen G et al. AcroBel - The Belgian registry on acromegaly: A survey of the "real-life" outcome in 418 acromegalic subjects. Eur J Endocrinol 2007; 157: 399-409

[24] Trouillas ], Sassolas G, Guigard MP et al. Relationships between pathological diagnosis and clinical parameters in acromegaly. Metabolism 1996; 45: 53-56
[25] Furuhata S, Kameya T, Otani M et al. Prolactin presents in all pituitary tumors of acromegalic patients. Hum Pathol 1993; 24: 10-15

[26] Tuna MM, Karakiliç E, Basaran MB et al. Immunostaining results of growth hormone secreting adenomas and their correlation with laboratory findings. Turkish Journal of Endocrinology and Metabolism 2016; 20: 69-71

[27] Kuhn E, Chanson P. Cabergoline in acromegaly. Pituitary 2017; 20 : $121-128$

[28] Trouillas ], Vasiljevic A, Lapoirie M et al. Pathological markers of somatotroph pituitary neuroendocrine tumors predicting the response to medical treatment. Minerva Endocrinol 2019; 44: $129-136$ 